UCRL-JC-126719

PREPRINT

\title{
Status of the PEP-II B-Factory High Energy Ring
}

U. Wienands, E. Reuter, P. Bellomo, E. Daly, A. Fisher, J. Gracia, A. Kulikov, N. Kurita, M. Pietryka, J. T. Seeman, T. Taylor, C. Belser, L. Bertolini, M. Mugge, J. Swan

This paper was prepared for submittal to the European Particle Accelerator Conference

Stiges, Spain

June 9-14, 1996

June 1, 1996

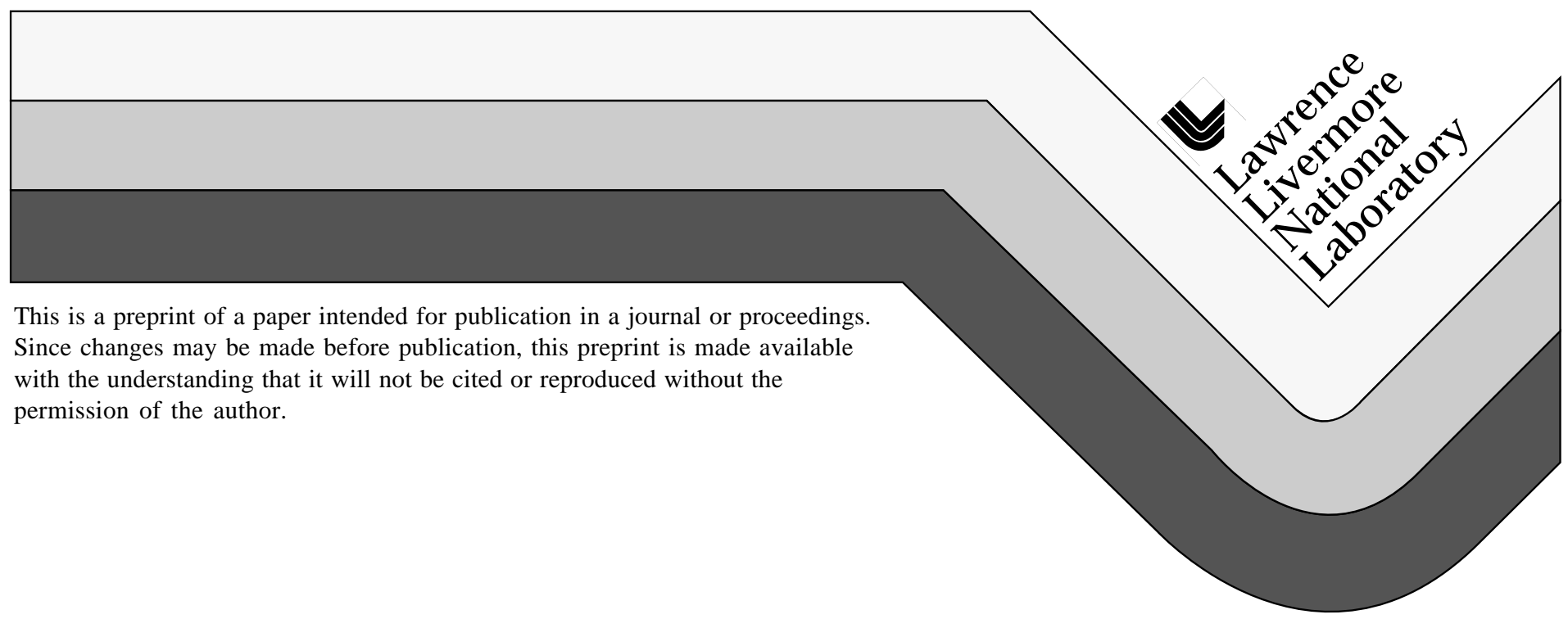




\section{DISCLAIMER}

This document was prepared as an account of work sponsored by an agency of the United States Government. Neither the United States Government nor the University of California nor any of their employees, makes any warranty, express or implied, or assumes any legal liability or responsibility for the accuracy, completeness, or usefulness of any information, apparatus, product, or process disclosed, or represents that its use would not infringe privately owned rights. Reference herein to any specific commercial product, process, or service by trade name, trademark, manufacturer, or otherwise, does not necessarily constitute or imply its endorsement, recommendation, or favoring by the United States Government or the University of California. The views and opinions of authors expressed herein do not necessarily state or reflect those of the United States Government or the University of California, and shall not be used for advertising or product endorsement purposes. 


\title{
STATUS OF THE PEP-II B-FACTORY HIGH ENERGY RING*
}

\author{
U. Wienands, E. Reuter, P. Bellomo, E. Daly, A. Fisher, J. Gracia, A. Kulikov, N. Kurita, \\ M. Pietryka, J.T. Seeman, T. Taylor; SLAC, Stanford, CA 94309; and \\ C. Belser, L. Bertolini, M. Mugge, J. Swan; LLNL, Livermore, CA 94550
}

\begin{abstract}
The $9 \mathrm{GeV}$ High Energy Ring (HER) of the PEP-II $B$ Factory is an electron storage ring under construction at SLAC. Significant progress has been made in the last year on all systems. As of mid 1996, all 192 dipoles have been installed, with installation of the quadrupoles underway. The vacuum system, for design currents up to $3 \mathrm{~A}$ average, is in production using a recently commissioned e-beam welder. Beam instrumentation systems are being fabricated. The interaction region will bring the HER beam into collision with the $3 \mathrm{GeV}$ beam of the Low Energy Ring; design of this section of the HER is in an advanced stage.
\end{abstract}

\section{INTRODUCTION}

The SLAC-LBL-LLNL PEP-II project is an asymmetric $3.1 \mathrm{GeV}$ on $9 \mathrm{GeV} \mathrm{e} \mathrm{e}^{+}-\mathrm{e}^{-}$collider designed for a peak luminosity of $\mathrm{L}=3 \times 10^{33} \mathrm{~cm}^{-2} \mathrm{~s}^{-1}$. In order to reach the design luminosity at moderate tune shifts (about 0.03 ) the circulating beam currents are large $-1 \mathrm{~A}$ in the HER and 2.1 A in the LER. A list of the important HER machine parameters is given in Table 1 . The facility is under construction in the PEP tunnel at SLAC $[1,2]$.

The magnet lattice of the ring is six-fold symmetric to fit the existing tunnel and has been described before [3]. The nominal beta function at the insertion point, $\beta_{y}{ }^{*}$, is $2 \mathrm{~cm}$; the lattice and the insertion quads support $\beta_{y}{ }^{*}$ as low as $1 \mathrm{~cm}$. The dynamic aperture of the lattice at $\beta_{y}{ }^{*}=1.5 \mathrm{~cm}$ is more than 125 .

Table 1. Top-level HER machine parameters.

\begin{tabular}{|ll|}
\hline Energy $(\mathrm{GeV})$ & 9 \\
Circumference $(\mathrm{m})$ & 2199.3 \\
Average beam current $(\mathrm{A})$ & 1 \\
Emittance, (horiz.,vert.) $(\pi \mathrm{nm}-\mathrm{r})$ & $(48,1.9)$ \\
Number of bunches & 1658 \\
Rf frequency $(\mathrm{MHz})$ & 476 \\
Beta functions at IP (x,y) $(\mathrm{m})$ & $0.5,0.02$ \\
Energy loss per turn $(\mathrm{MeV})$ & 3.69 \\
\hline
\end{tabular}

\section{ACCELERATOR SYSTEMS}

\section{Installation}

As of Oct. 1995 all 192 refurbished PEP dipoles have been reinstalled in the tunnel. Quadrupoles are being assembled and prealigned on rafts together with their

\footnotetext{
* Work supported by US Dept. of Energy under contracts DE-AC03-76SF00515, DE-AC03-76SF00098, DE-AC0381ER40050, DE-AC03-76ER70285 and W-7405-Eng-48.
}

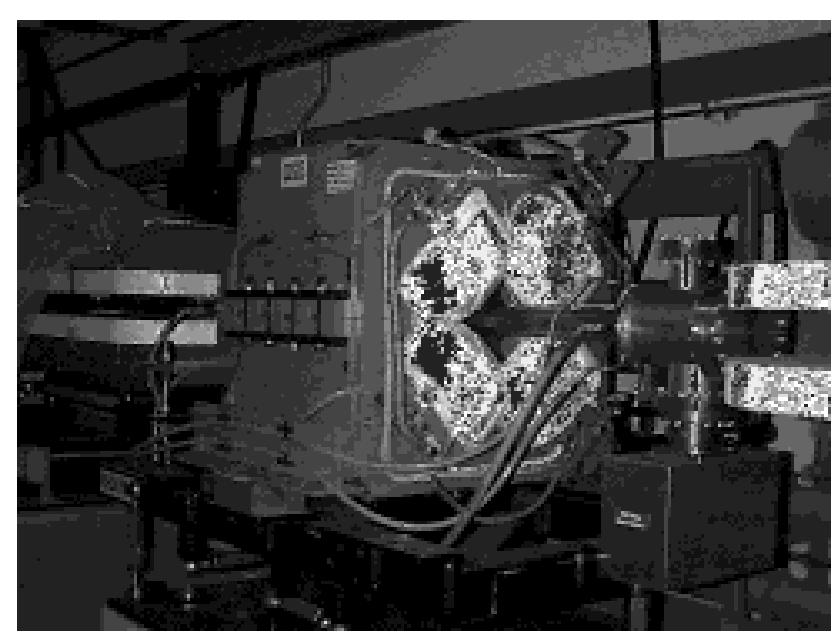

Figure 1. Quadrupole raft installed in the PEP Tunnel

associated orbit correcting dipoles and sextupoles where applicable. The completed rafts are then transported to the tunnel where they are positioned and surveyed (Fig. 1). The quadrupoles are sorted by their integral gradient to match members of a family powered by one supply as closely as possible. Surveying data gathered so far indicates that the rafts are being installed to within $1 \mathrm{~mm}$ rms of their final position before alignment. A slight setback was encountered when it became evident that the fixtures used as quadrupole fiducials to locate the center of each magnet did not give reproducible results. Now each quadrupole is fiducialized individually.

Quadrupole vacuum chambers (which are captive to the magnets) are installed in the quadrupoles at raft assembly, before installation in the tunnel. Dipole vacuum chambers are installed in the tunnel as they slide easily into the opening of the C-magnets.

It had been decided early on in the project to reuse the existing cable plant as much as reasonable, therefore, a significant amount of cabling already exists in place but significant effort is required to reconfigure the cabling for the different configuration of the HER magnets and installation of new cables for the LER. This activity is scheduled for completion by the end of 1996 .

\section{Magnets}

A fraction of the HER quadrupole magnets are newly built, using the same lamination dimensions as the existing quadrupoles. The laminations for these magnets have been obtained from a local vendor, while the coils have been built at BINP, Novosibirsk. Fabrication of these magnets is about halfway complete, with 
completion scheduled for August 1996. Magnetic measurements of the first assembled magnets have confirmed that the magnetic properties differ only insignificantly from those of the existing quadrupoles.

\section{Vacuum System}

The HER arc vacuum system is a copper system designed to absorb the $10 \mathrm{MW}$ synchrotron radiation power generated by a $9 \mathrm{GeV}, 3 \mathrm{~A}$ beam. An electronbeam welder has been procured to allow welding of the up to $5.8 \mathrm{~m}$ long $\mathrm{Cu}$ chambers under clean-room conditions. Production of the dipole chambers involves several steps: Welding of the cooling bars, stretchforming across a mandrel to follow the curvature of the beam orbit in the dipoles, welding of the screen separating the distributed ion pump (DIP) from the beam channel, inserting the DIP and finally welding of the flanges and end-pieces of the DIP channel. The dipole chambers are glow-discharge cleaned before insertion of the DIP and the complete chamber is baked at $200{ }^{\circ} \mathrm{C}$ before delivery to the tunnel. Measurements of the pumping speed of the first assembled dipole chamber have shown a net pumping speed of approx. $180 \mathrm{l} / \mathrm{s} / \mathrm{m}$ at $10 \times 10^{-9}$ torr. Fabrication of the quadrupole chambers follows a somewhat similar procedure. All chambers are in full production and are being installed in the tunnel.

The straight-section vacuum system does not have to absorb much synchrotron radiation power and is therefore made of stainless steel tubing with water cooling. All components are in production in the SLAC workshops, while cleaning is done in a facility built at LLNL.

Prototypes of arc and straight-section bellows have been tested for $\mathrm{rf}$ heating and trapped modes. The tests have been successful: no evidence for excessive heating has been found in the straights bellows under rf power (at $480 \mathrm{MHz}$ ) calculated to exceed the level expected at $3 \mathrm{~A}$ beam current; also, no evidence of trapped modes below cut-off of the vacuum system has been found. The straights bellows is described in detail in [4].

\section{Beam Diagnostics}

The suite of beam diagnostics under construction for PEP-II is described in more detail elsewhere.[5]

Construction of the beam position monitor (BPM) system is progressing on schedule. The $15 \mathrm{~mm}$ pickup buttons are welded into the quadrupole chambers during fabrication; each chamber is subsequently calibrated at the BPM frequency of $952 \mathrm{MHz}$. There are a number of additional BPM for special-purpose diagnostics.

An extensive system of beam-loss monitors (BLM) is in design in order to allow localization of beam losses and to provide a large dynamic range-about $10^{6}$-in lossdetection. The detector element is a Cherenkov counter equipped with a subminiature PMT; the whole detector unit can easily be mounted close to the beam line. Lead shielding $(10 \mathrm{~mm})$ is provided against synchrotron radiation. All electronics are contained in CAMAC modules located underneath selected dipoles for shielding against radiation.[6] This system also provides a signal to the beam-abort trigger system (BATS).

The dc current transformer (DCCT) uses a commercial unit by Bergoz. The housing is based on a design used successfully at the ALS and has been built. A current resolution of $5 \mu \mathrm{A}$ allows for the fast, on-line beam-lifetime calculations essential for tuning the machine.

The bunch-current monitor (BCM) uses the signal from a dedicated set of BPM buttons, which are summed for this purpose. High-speed conversion using an 8-bit, $500 \mathrm{MHz}$ DAC, parallel processing and accumulation of the data during one injection cycle yield an effective resolution of about $10 \mathrm{bit}$; essential for achieving a resolution of better than $0.5 \%$ for the charge measurement of a single bunch during one injection cycle $(17 \mathrm{~ms})$. The BCM will be calibrated against the DCCT.

The synchrotron light monitor is challenging because of the very high synchrotron radiation flux from the ring dipoles. The first mirror has to be able to withstand this flux without significant distortion on a $10 \mathrm{~nm}$ scale. This challenge is being met by adopting a very shallow angle of incidence- $4^{\circ}$ - at the first mirror. A $4 \mathrm{~mm}$ high slot in the mirror lets the X-ray fan, with its much narrower vertical spread and much higher intensity, pass through to a separate dump thermally decoupled from the mirror.[7]

\section{THE INTERACTION REGION}

PEP-II is designed for head-on collisions in order to avoid issues arising from synchro-betatron coupling. Due to the need to separate the beams quickly to avoid parasitic crossings, permanent-magnet dipoles are located within the detector. The innermost quadrupole, again a permanent magnet, acts on both the HER and the LER beams. Outside of this quadrupole the magnetic elements are separate for each ring. One of the HER insertion quadrupoles (Q4) will be offset both vertically and horizontally to compensate for the bending of the asymmetric and tilted detector solenoid.[8]

During initial HER commissioning, the shared magnets will not be present. The HER insertion quadrupoles Q4 and Q5 (Fig. 2) will run at higher currents to provide the required focusing. By coincidence, the increased strength in Q4 will also just compensate for the missing dipoles. The commissioning lattice is then tuned such that the beam sizes outside the insertion quadrupoles are identical to those in the final configuration; in this way, background measurements that will make up an important part of the commissioning activities in 1997 can be performed with a configuration very close to the final one after installation of the LER and the detector.

Both HER insertion quadrupoles are laminated ironcopper magnets. Design challenges arise from the large 


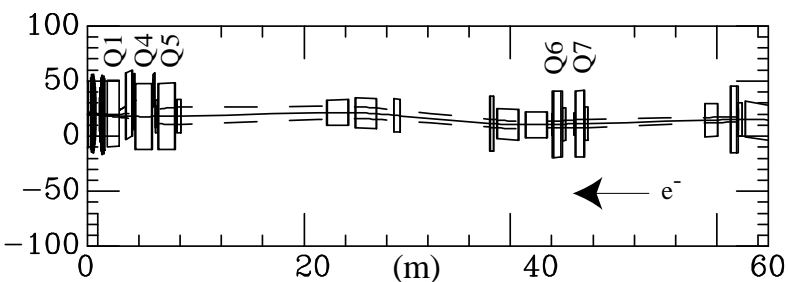

Fig. 2. Layout of the upstream HER Interaction Region

beam size in these magnets and the small separation of the beams at these quads, restricting the room for the magnet coils. Field-uniformity requirements are tight: non-linear field harmonics are not to exceed $10^{-4}$ of the quadrupole field at a radius of $59 \mathrm{~mm}$.

For QF5, the outer one of the insertion doublet, the cores of existing PEP insertion magnets are reused. A channel is cut into the side of the yoke for the LER; an insert restores the mechanical integrity of the magnet. New coils are being built that occupy significantly less space than the original coils. POISSON calculations done for both the original and the new configuration confirm that the effect of these modifications on the magnetic field harmonics is small. The shape of the end chamfers will be iterated in order to keep the integral field harmonics at or below the specifications.

At QD4 the two beams are even closer together $(15 \mathrm{~cm}$ separation). There are additional restrictions on the outside dimensions of the magnet due to its being partially within the detector envelope. A satisfactory coilwindow was found by letting the coil protrude against the vacuum chamber. Field distortions in the vicinity of the coil are avoided by letting the innermost layer of the coil follow the field lines; this is essential in keeping the current density reasonable.

For both magnets, stray fields on the LER axis have been calculated to be a few Gauss. Iron or $\mu$-metal shields around the LER vacuum pipe will reduce these fields by about one order of magnitude to acceptable levels.

The vacuum in the IR upstream of the detector is crucial for low-background operation of the facility. NEG pumping will be provided throughout the critical region between the low-field bends and the detector to achieve an average pressure of $\leq 0.5$ nTorr (Fig. 3). Rather than using NEG strips, the pumps will consist of NEG discs stacked on a $6 \mathrm{~mm}$ tube, which in turn houses the heater to regenerate and activate the NEG. Thus, pumping speed and capacity of the pump are enhanced by an order of magnitude compared to simple strips. Care is being taken to ensure the NEG material is as free of magnetic contaminants as possible. The vacuum chambers are based

on the same $\mathrm{Cu}$ extrusions as used in the HER arcs.

\section{BEAM INJECTION AND ABORT}

The Linac beam is injected vertically into the PEP-II rings. A horizontally bending Lambertson septum and a vertically bending current-sheet septum act on the

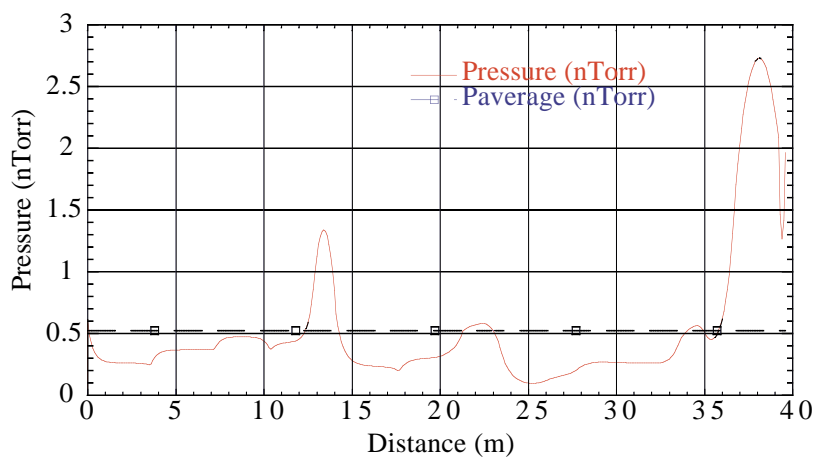

Fig. 3. Pressure profile, upstream interaction region

injecting beam to steer it horizontally onto the ring axis and vertically inject at nominally $8.5 \sigma_{y}$. A local dc bump displaces the ring orbit so that the $10 \sigma$ beam envelope touches the ring side of the septum; two fast kickers pulse the orbit by another $4 \sigma$ to receive the next linac pulse. The kickers are at $180^{\circ}$ phase advance relative to each other and thus produce a localised bump as well. The dc bump with four vertical dipole magnets allows adjustment of position and injection angle to optimize the injection.

At $3 \mathrm{~A}$ beam current, the stored energy in the HER is about $200 \mathrm{~kJ}$. Such large energies can cause damage to the vacuum system if dumped in the ring. The beam abort system resides just upstream of the injection area. A kicker magnet will deflect the beam onto a beam dump located underneath the injection Lambertson septum. In order to protect the exit window and the dump itself, the kicker sweeps the beam vertically across an about $15 \mathrm{~mm}$ long stripe. The beam dump itself uses layers of $6 \mathrm{X}_{0} \mathrm{C}$, $5 \mathrm{X}_{0} \mathrm{Al}$ and $12 \mathrm{X}_{0} \quad \mathrm{Cu}$ in a water-cooled aluminum housing.

\section{REFERENCES}

[1] J.T. Seeman et al., "PEP-II Status and Plans," Proc. IEEE Part. Accel. Conf., Dallas, TX, May 1995, p. 486.

[2] U. Wienandset al. , "Status of the High Energy Ring of the PEP-II $B$-Factory", ibid., p. 530.

[3] M.H. Donald et al., "Lattice Design for the High Energy Ring of the SLAC B-Factory," ibid., p. 591.

[4] C. Belser, J. Berget al. , "High Current Rf Shield for PEP-II Vacuum Expansion Joint," these proceedings.

[5] A. Fisher et al., "Diagnostics Development for the PEP-II $B$-Factory," Proc. Beam Instr. Workshop, Argonne, Il, May 6-9, 1996, to be published.

[6] J. Liu et al., "Calculations of the Radiation Doses to the Electronic Equipment in the PEP-II Tunnel," NIM A 359, 463 (1995).

[7] A. Fisher et al., "Design of the Synchrotron-Light Monitor for PEP-II," these proceedings.

[8] L. Bertolini, M. Sullivan et al., "Interaction Region Design at the PEP-II $B$-Factory," these proceedings. 


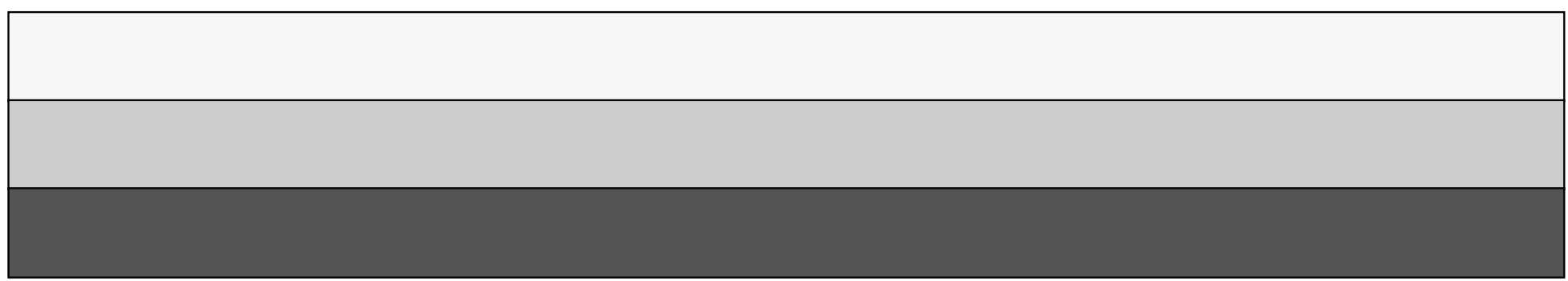

